\title{
Watermarking, a Mature Technology - Retrospect and Prospect
}

\author{
Bede Liu \\ Princeton University, USA \\ liu@Princeton.EDU
}

\begin{abstract}
Digital Watermarking has been proposed to manage digital rights, for authentication, to recover lost information, to monitor performance, and other applications. The efforts of many researchers from different disciplines have helped us to understand the basic issues and the challenges, and to help guiding the community toward actual applications. As digital watermarking matures as a technology, it is time to review what has been accomplished and to speculate what may be expected. In this talk, we will try to highlight the key development in digital watermarking, to examine the effectiveness in some applications, and to offer some thoughts regarding future development of digital watermarking.
\end{abstract}

